\title{
COVID-19, PHYSICAL DISTANCING IN THE WORKPLACE AND EMPLOYEES' MENTAL HEALTH: IMPLICATIONS AND INSIGHTS FOR ORGANIZATIONAL INTERVENTIONS - NARRATIVE REVIEW
}

\author{
Salima Hamouche \\ Faculty of Management, Canadian University Dubai, Dubai, UAE \\ revised: 11.1.2021; \\ accepted: 18.1.2021
}

received: 16.11 .2020

\begin{abstract}
SUMMARY
Physical distancing is one of the non-pharmaceutical measures adopted to prevent the spread of COVID-19. Although it appears to be effective in mitigating this spread, its implementation in workplaces may undermine employees' mental health. In fact, torn between the fear of contagion and the need to maintain their jobs, employees must also comply with physical distancing measures in the workplace, which alter social interactions and set a predetermined frame and distance that guide employees' behaviors within the organization while they need empathy. This situation might increase their level of stress.

This paper is a narrative review that addresses the impact of physical distancing in the workplace on employees' mental health. It presents the main factors that might moderate this impact and it recommends organizational interventions that can help to mitigate it.

Physical distancing measures in workplaces are necessary and inevitable. Notwithstanding, they might undermine employees' mental health, whence the importance to implement proper organizational actions to support employees and to facilitate their adaptation, in this unprecedented organizational change.

This paper examines a relatively unexplored topic. It goes beyond examining social isolation to explore how setting a predetermined frame and distance can have an impact on employees' mental health and recommends interventions that might help organizations to prevent mental health issues.
\end{abstract}

Key words: COVID-19- occupational health - social distancing - mental health - human resource management

$$
* * * * *
$$

\section{INTRODUCTION}

Declared a global pandemic on March 11, 2020, by the World Health Organization (WHO 2020c), COVID19 emerged in the city of Wuhan, China in December 2019, has spread at a lightning speed to affect the whole world.

Containing and controlling the transmission of COVID-19 was challenging for the whole world, considering the absence of a vaccine (Williams et al. 2020). In this context, many countries have implemented emergency control measures to reduce the risk of the virus spread, e.g. schools, and universities were closed, borders were closed, flights were canceled as well as public and social events (Yip \& Chau 2020).

Trying to recover from this economic shock, businesses and workplaces have reopened, in the mid of the ongoing COVID-19 crisis, under extraordinary rules and a new functioning (Galea et al. 2020) that no one can predict when it will end. Besides physical guards and individual protective equipment (e.g. to wear masks, and to use sanitizer) (Shaw et al. 2020), physical distancing also known as social distancing is one of the forms of non-pharmaceutical physical measures required in the workplaces (WHO 2020a). Despite their recognized effectiveness in reducing the transmission of COVID-19 (Williams et al. 2020), physical distancing measures may negatively impact employees' mental health (Williams et al. 2020, Fischer et al. 2020, Zhang et al. 2020b). Indeed, during a pandemic, individuals may change their behavior to protect themselves and their beloved ones from an infectious disease, however, it can be psychologically heavy to bear and to sustain, even if they may know that it is necessary and inevitable to protect themselves.

According to some authors, the unprecedented scale and severity of physical distancing measures that have been implemented in response to COVID-19 are likely to generate an unprecedented scale and severity in terms of psychological impact on individuals (Williams et al. 2020, Fischer et al. 2020). The studies on the impact of physical distancing on employees' mental health are sparse. Some of the studies focus mainly on the effectiveness of these measures in the reduction of virus transmission and spread (Regmi \& Lwin 2020), while little is known about the impact of physical distancing on individuals' mental health (Zhang et al. 2020b, Galea et al. 2020), mainly in the workplace. It is necessary to examine this impact considering the possibility that these movement restrictions remain momentarily to prevent virus spread (Fischer et al. 2020).

This narrative review aims to address the impact of physical distancing in the workplace on employees' mental health and to present the possible moderators of this impact as well as organizational interventions that might mitigate this impact. 


\section{PHYSICAL DISTANCING OR SOCIAL DISTANCING}

Physical distancing named in most cases "social distancing (Galea et al. 2020, Centers for Disease Control and Prevention 2020) is one of the mitigating non-pharmaceutical physical measures adopted and currently used by most of the countries to prevent COVID-19 transmission. "Physical distancing" and "social distancing" are often used in an interchangeable way (Gupta \& Wong 2020) to refer to the deliberate increase of the physical distance between people to prevent the spread of illness (Prin \& Bartels 2020). To practice social or physical distancing, individuals need to stay at least 6 feet (about 2 arms' length) from other individuals (Centers for Disease Control and Prevention 2020, WHO 2020a). Physical distancing can be implemented within contained (e.g. offices, homes), shared (e.g. cafeteria), and natural spaces (e.g. parks) (Prin \& Bartels 2020). It is different from self or group quarantine and isolation. Quarantine refers to limiting the movement of asymptomatic individuals who have been exposed to the virus, whereas isolation refers to keeping individuals with identified infection away from other people (Prin \& Bartels 2020).

In the present paper, we retained the concept of "physical distancing" instead of "social distancing" for three main reasons: firstly, the main objective of this review is to examine the practices of physical distancing in the workplace and to analyze their impact on employees' mental health. Secondly, according to Gupta \& Wong (2020, p.1) "social distancing is a misnomer. It is not self-explanatory, conceptually ambiguous, practically misleading, and intellectually misplaced." Mainly because "practices of social distancing are purely physical-spatial with nothing social in nature" (Gupta \& Wong 2020, p.1), finally, the use of ICT (information communication technologies) and social media network does not seem to leave room for discussion about the social distance but rather physical distance. Therefore, researchers call for being careful about the interchangeability of the use of these two concepts, to avoid misleading individuals and driving them indirectly toward social isolation (Gupta \& Wong 2020). Individuals need to be encouraged to maintain social interactions, through ICT and social media while respecting physical distancing measures (Zhang et al. 2020b) and to avoid isolation and loneliness that may undermine their mental health.

\section{PHYSICAL DISTANCING IN THE WORKPLACE}

Physical distancing is among the universal prevention measures that organizations are required to implement in the workplace to prevent the spread of COVID-19
(WHO 2020a, Cirrincione et al. 2020). In this case, based on WHO (2020a) guidelines, they need to introduce measures to keep a distance at least 1 meter between individuals and to avoid direct physical contact between them (e.g. handshaking, touching); to keep a physical distance at least 1 meter apart from work area and common spaces, like stairs, lifts, canteens and queuing of employees, visitors, and customers. They are also required to reduce the number of employees who need to be physically present in the company based on the size of the workplace (no more than 1 person per every 10 square meters) (Cirrincione et al. 2020, WHO 2020a) In this context, they should implement shift, teleworking or split-team arrangements. Organizations should also avoid crowding of employees at common spaces by managing their working hours, minimizing physical meetings, and optimizing the use of virtual meetings.

It is possible to identify three types of physical distancing in the workplace: (1) teleworking (Mustajab et al. 2020), (2) static physical distancing, and (3) dynamic physical distancing (Cristani et al. 2020). In teleworking, the employee is required to be physically far from the workplace and to continue working from home if the nature of his job allows it (Cockburn 2020). However, not all job positions can be performed from home. Thus, employees whose job tasks cannot be performed remotely are required to be physically present in the workplace. In this case, two types of physical distancing will be applied: static and dynamic distancing. Static physical distancing refers to the physical workspace, mainly the proximity between employees while they share the same workspace, e.g. office and production line. Whereas, dynamic physical distancing refers to the movement (El Husseiny \& El Husseiny 2013) of employees within the workplace, where they need to move while respecting a distance of at least 6 feet (about 2 arms' length) from other employees. The complexity of implementing physical distancing in the workplace may vary based on the number of employees and the size of the workspace.

Although physical distancing measures appear to be effective in mitigating the expansion of COVID-19, they may lead to an increase in mental health issues (Fischer et al. 2020).

\section{PHYSICAL DISTANCING IN THE WORKPLACE AND EMPLOYEES' MENTAL HEALTH}

COVID-19 is associated with a high level of mental health issues (Ćosić et al. 2020, Jakovljevic 2020, Qiu et al. 2020, Rajkumar 2020). Furthermore, studies related to social distancing during COVID-19 pointed out a negative impact of these measures on individuals' mental health (Galea et al. 2020) (e.g. depression, anxiety, and psychological distress) (Williams et al. 2020). 
According to some authors, besides hospitals, workplaces can be setting of virus transmission risk (Tan et al. 2020). In this context, COVID-19 creates a new workplace hazard (Hecker 2020) that may represent a significant source of stress for employees (Shaw et al. 2020). Torn between the pressure to keep their job and their fear of being contaminated, returning to work may not be an easy decision for employees, especially when the return to work occurs in an uncertain environment (Shaw et al. 2020). Return to work is a necessity, but it can be a source of discomfort and anxiety for employees. The study of Balkhi et al. (2020) showed that due to COVID-19, individuals are apprehensive of leaving their house, while they feel anxious if it is a member of their family who has to leave instead.

Thereby, the physical distancing measures implemented in workplaces may represent another source of stress for employees. It is an atypical practice (Zhang et al. 2020b) both inside and outside the organization, where individuals are required to act differently than in times of non-crisis (Jin et al. 2020). In these conditions, individuals may perceive a loss of structure and routine (Williams et al. 2020). Undoubtedly, COVID-19 is an unprecedented crisis for workplaces. It has greatly changed work methods and human interaction in organizations (Mustajab et al. 2020). In this context, physical distancing measures represent an organizational challenge that needs to be addressed. It might raise the question of how to maintain simultaneously physical distance and emotional closeness (Yip \& Chau 2020). Indeed, social ties are important for the emotional stability of the individual (Aleman \& Sommer 2020). "Humans are social beings" (Carvalho Aguiar Melo \& De Sousa Soares 2020, p.1), who need to continuously interact with each other, to simply exchange ideas, states of mind or to achieve their goals (Cristani et al. 2020). On this basis, the time spent in physical proximity with others is crucial for individuals' development and psychological stability (Cristani et al. 2020), which may suggest that measures that alter their social interactions or foster their feeling of social isolation are likely to increase their anxiety, depression and psychological distress (Carvalho Aguiar Melo \& De Sousa Soares 2020). In workplaces, employees need to follow the physical distancing measures. Besides keeping a distance of at least 1 meter from others, they need to avoid direct physical contact with other persons, i.e. to avoid hugging, touching, shaking hands (WHO 2020a, Cirrincione et al. 2020). With these measures, individuals miss the freedom and the choice to interact with others at any distance (Mehta 2020), which may suggest that they cannot behave spontaneously, they need to calculate and to control their behaviors within predetermined frame and conditions while interacting with other people, e.g. with their friends in the workplace, which can represent a psychological burden for them. Furthermore, work from home may be psychologically challenging for individuals who need to restructure their work patterns and to balance their professional and private life while working from home (Williams et al. 2020).

The study of Bryan et al. (2020) conducted in the early stages of COVID-19 showed that physical distancing measures like working from home and large gathering bans have no impact on an individual's mental health, like depression and suicide attempts. However, the authors argued that their survey was fielded during the first few months of the pandemic. Probably the deleterious effects of physical distancing on mental health outcomes had not yet emerged (Bryan et al. 2020). In fact, according to the study of Zhang et al. (2020b), the duration of social distancing can explain the impact of this measure on individuals' mental health. The results of this study suggest that long-term social distancing leads to increasingly negative moods, which can negatively impact the individual's mental health. However, it is not the case for short-term distancing. For example in the initial stages of social distancing, this study showed that negative mood has declined and individuals' resilience has increased, probably due to the time spent with the family at home and the use of virtual social contacts (e.g. via Zoom), which brought people together virtually. This can be applied to employees working from home, but it is not the case for employees who are required to be physically present in the workplace. Still very little is known about the impact of physical distancing on their mental health while some authors suggested that physical distancing measures put individuals in a situation where they are deprived of their freedom, their habits, and their routine which may lead to a high risk of mental health issues like depression and anxiety (Venkatesh \& Edirappuli 2020). Other authors pointed out that restricting close human connections may soon involve mental health issues, like depression and anxiety (Abel \& McQueen 2020).

\section{Factors that may mitigate or aggravate the impact of physical distancing on employees' mental health}

Three main factors may moderate the impact of physical distancing on employees' mental health: individual, organizational and societal factors.

\section{Individual factors}

The implementation of physical distancing in the workplace requires a modification of work habits (Shaw et al. 2020). Employees change their behavior in an attempt to protect themselves and their beloved ones from infectious diseases as well as to comply with the organizational policies and measures. Organizations need to take into consideration the fact that employees may have different reactions. Indeed, the comfort level in such circumstances may vary from one individual to another depending on factors specific to each employee. 
Besides, the perception of safety, threat, and risk of contagion can vary from one individual to another (Hamouche 2020). Some employees are eager to return to their physical workplace while others are worried about being infected and are apprehensive to leave their homes (Zhang et al. 2020a). Moreover, most of them were quarantined and some of them have been infected or have indirectly suffered from this virus (Brooks et al. 2020), which may suggest that they are already psychologically vulnerable (Shaw et al. 2020), considering that quarantine during a pandemic is associated with poorer mental health (Brooks et al. 2020), with a high prevalence of symptoms of psychological distress and disorder (Wang et al. 2020). Some studies showed that individuals with pre-existing mental health illness may suffer more compared to other individuals from the limitation of their interpersonal interactions (Venkatesh \& Edirappuli 2020). It is also the case of individuals with higher levels of anxiety, who may be more likely to overreact toward the adopted policies (Jin et al. 2020).

Furthermore, according to some authors, organizations need to pay more attention to vulnerable groups such as the elderly, women, and migrant workers (Qiu et al. 2020). The study of Atchison et al. (2020) showed that the adoption of social-distancing measures was higher in those aged over 70 compared to younger adults aged 18 to 34 years. The elderly belong to the main target group of physical distancing considering their physical vulnerability, thus, they need significant psychological support (Vieira et al. 2020). Individuals living alone are also considered as a high-risk population in a period of a pandemic, during the implementation of physical distancing (Aleman \& Sommer 2020). Besides, some authors argued that women can react more intensively to stress related to COVID-19 (Zhu et al. 2020).

\section{Social factors}

Social exclusion and social stigma are the most common social outcomes of a pandemic (Xiang et al. 2020, Jakovljevic et al. 2020). Fear of contagion may lead individuals to develop a form of stereotyping, by associating COVID-19 to a specific category of people mainly those related to the disease epicenter (Xiang et al. 2020); survivors, their relatives (Zhang et al. 2020a), and people who have been in contact with other people who have been quarantined (Brooks et al. 2020). In this case, individuals may avoid them, blame them, or spread misleading rumors about them. Targeted individuals may feel rejected, isolated, and discriminated which may undermine their mental health (Brooks et al. 2018) and challenge their capacity to bear physical distancing in the workplace. Stigmatization may lead to a high level of psychological distress and depression (Zhang et al. 2020a). Hence, coupled with physical distancing measures, it may worsen the relationship between employees in the workplace, in the context of physical distancing.

\section{Organizational factors}

Organizational factors may refer to the occupational role and the sector of activity. The risks and exposure to a pandemic generally vary based on the work environment and the employee's occupational role (Brooks et al. 2018). Physical distancing in the hospital represents an important measure of protection for healthcare workers (Prin \& Bartels 2020). This measure may be simple in theory but very challenging in practice for employees who used to work together closely, for example in the case of nurses, anesthesiologists, surgeons (Prin \& Bartels 2020). This context may suggest that these measures will be an additional source of stress for these employees who are already physically and psychologically exhausted because of their workload and the very high exposure to the virus (Xiang et al. 2020).

\section{ORGANIZATIONAL INTERVENTIONS TO MITIGATE THE NEGATIVE IMPACT OF THE WORKPLACE PHYSICAL DISTANCING ON EMPLOYEES' MENTAL HEALTH}

According to some authors, the likelihood is that COVID-19 will become endemic, which requires longterm behavioral adjustment (Regmi \& Lwin 2020). This suggests that organizational interventions should be oriented toward the short and the long-term, to be able to sustain the company's business while protecting employees. Mitigating these mental health issues requires proper and relevant organizational interventions, that may be applicable in the current pandemic and even beyond. There are guidelines for workplaces to prevent the spread of the virus (WHO 2020a, Cirrincione et al. 2020, Cockburn 2020). However, we do not know much about potential organizational interventions that mitigate the impact of physical distancing in the workplace on employees' mental health. In this section, we recommend some organizational interventions from a human resource management perspective that may help to mitigate this impact.

\section{Management of the Physical distancing in the workplace}

Effective management of COVID-19 can help to reduce individuals' anxiety (Shaw et al. 2020). Organizations should properly and effectively manage the physical distancing in their workplaces, by making a distinction between measures applied for employees who work from home and those applied for employees who are physically present in the workplace.

For the employees who work from home, organizations should help them to overcome the challenges related to teleworking by providing the proper technological equipment that facilitates their work and to train 
them on the use of this equipment, which will reduce their level of stress. They should also assist them in setting healthy boundaries between their work and their private life, by keeping continuous communication about the expectation related to their work, and to provide flexibility for those who need to take care of their kids or elderly (Cockburn 2020).

For the employees who are required to be physically present in the workplace, organizations need to implement clear guidelines related to the protection measures including physical distancing. According to Cristani et al. (2020), it is important for the organization to identify the required geometric space and to make it simple and easy to understand for employees, which may reduce their stress and anxiety e.g. clear marking on the floor and barriers (WHO 2020a). Organizations need to provide the required protection material for those who need to be present in the workplace (e.g. Masks, Sanitizers, etc), and to post prevention guidelines (e.g. wash hands, avoid touching eyes). Moreover, organizations need to carry out a regular risk assessment for work-related exposure to COVID-19 (WHO 2020b). In this context, employees' involvement is needed as well as (Cockburn 2020) partnership with occupational health services and health authority (WHO 2020b).

\section{Communication and training}

Communication, interaction, and workgroup interdependence represent the main characteristics of individuals working together. Continuous clear communication should help to build trust with employees (Williams et al. 2020) and reduce their level of stress and anxiety. According to Vieira et al. (2020) uncertainty generated by COVID-19, if combined with mobility restrictions may increase the individual's feeling of insecurity, anxiety, emotional strains, and psychiatric disorder (Vieira et al. 2020). Without proper information, individuals may start speculation, which may lead to negative emotional contagion that may accelerate the spread of rumors and misinformation (Jin et al. 2020). In this context, information and training play a crucial role in increasing the awareness of employees about the necessity and the importance of physical distancing in the workplace, and safe operating practices (Hecker 2020).

Occupational health and safety guidelines, related to the reopening of the workplace, need to be communicated to employees (Shaw et al. 2020) before their return to work. They should receive a picture or a chart with the main changes related to the physical distancing to visualize the main changes that have occurred in their working space (Cristani et al. 2020). Withal, organizations should provide clear information about the purpose of the physical distancing measures, their impacts on work organization, the expected behaviors, their main challenges, and the available resources to support the employees.
Besides, according to some authors, providing accurate and timely information about the disease and training may minimize stigmatization (Cristani et al. 2020), and prevent the development of mental health, mainly among employees who can be a target of this stigma.

\section{Preparedness and planning of return to work}

Employers should develop a return-to-work plan, before bringing back employees physically to their workplace after a period of quarantine or teleworking (Hamouche 2020, Rueda-Garrido et al. 2020), which may help to reduce their level of stress and prevent the risk of mental health issues. They need to adapt this plan to their context and to establish their own pace by bringing back employees gradually to the workplace. In this context, a discussion between the manager and the employee about the company's expectations and plans should help to prepare the employees for a smooth return back to the workplace, and to identify employees who need to get accommodation and a gradual return to work (Durand et al. 2014) instead of bringing them back while they are not yet psychologically or physically ready for it, especially in the case of employees who have been contaminated or who have suffered from a mental health illness during the lockdown and quarantine (Hamouche 2020).

\section{Social support}

Social support at work is recognized and largely documented in the literature as a buffer of mental health issues in the workplace. Some studies showed that inadequate psychological support from the employer represents a risk factor for poor mental health (Brooks et al. 2018). Thus, it is important for employers to develop and to implement mental health support programs and services for employees when they return to their work, to minimize their potential anxiety and stress as well as mental health issues (Tan et al. 2020, Xiang et al. 2020). In these circumstances, employee assistance programs should be offered, for all organization's members (Hamouche 2020).

Some authors suggested that the use of digital technologies is likely to bridge social and physical distancing (Galea et al. 2020, Zhang et al. 2020b). Organizations can create virtual workplaces that foster collaboration and connection between employees to reduce their loneliness (Galea et al. 2020) and strengthen their ties in the workplace. In this context, organizations may offer their employees the possibility to have access to telemedicine mental health services, which include mental health counseling and virtual visits (Galea et al. 2020, Yip \& Chau 2020). Some authors recommend strengthening and improving the accessibility to these programs and services, especially after reviewing the initial coping and management of COVID-19 (Qiu et al. 2020) These types of interventions can be included in employees' assistance programs. 
The development of a supportive environment in the workplace (Brooks et al. 2018) and the mobilization of relevant resources for emotional support help to enhance resilience during pandemics and to mitigate the negative impact of social isolation feeling and the fear of contagion on employees (Brooks et al. 2018). Social support at work can be fostered by developing and maintaining continuous communication with employees, e.g. through the organization of regular virtual team meetings. Some authors suggested to be disciplined in physical distancing but at the same time to be creative in how to maintain this emotional closeness (Yip \& Chau 2020).

\section{CONCLUSION}

Physical distancing measures in workplaces are necessary and inevitable. Notwithstanding, they might undermine employees' mental health, whence the importance to implement proper organizational actions to support employees, to facilitate their adaptation to this unprecedented organizational change, and to protect their mental health.

\section{Acknowledgements: None.}

\section{Conflict of interest: None to declare.}

\section{References}

1. Abel T \& Mcqueen D: The COVID-19 pandemic calls for spatial distancing and social closeness: not for social distancing! Int. J. Public Health 2020; 65:231-231

2. Aleman A \& Sommer I: The Silent Danger of Social Distancing. Psychol Med 2020; 1-8

3. Atchison C J, Bowman L, Vrinten C, Redd R, Pristera P, Eaton $J W$ et al.: Perceptions and behavioural responses of the general public during the COVID-19 pandemic: A cross-sectional survey of UK Adults. medRxiv 2020;1-21

4. Balkhi F, Nasir A, Zehra A \& Riaz R: Psychological and Behavioral Response to the Coronavirus (COVID-19) Pandemic. Cureus 2020; 12: e7923

5. Brooks SK, Dunn R, Amlôt R, Rubin GJ \& Greenberg N: A Systematic, Thematic Review of Social and Occupational Factors Associated With Psychological Outcomes in Healthcare Employees During an Infectious Disease Outbreak. J Occup Environ Med 2018; 60:248-257

6. Brooks SK, Webster RK, Smith LE, Woodland L, Wessely $S$, Greenberg $N$ et al: The psychological impact of quarantine and how to reduce it: rapid review of the evidence. The Lancet 2020; 395:912-920

7. Bryan CJ, Bryan AO \& Baker JC: Associations among state-level physical distancing measures and suicidal thoughts and behaviors among U.S. adults during the early COVID-19 pandemic. Suicide Life Threat Behav 2020; e12653:1-17

8. Carvalho Aguiar Melo M \& De Sousa Soares D: Impact of social distancing on mental health during the COVID-19 pandemic: An urgent discussion. Int $J$ Soc Psychiatry 2020; 66: 625-626

9. Centers for disease control and prevention: Social Distancing. Keep Your Distance to Slow the Spread [Online]. USA: CDC 2020. (Accessed 07-06-2020). Available at: https://www.cdc.gov/coronavirus/2019-ncov/preventgetting-sick/social-distancing.html

10. Cirrincione L, Plescia F, Ledda C, Rapisarda V, Martorana D, Moldovan $R$ E et al.: COVID-19 pandemic: Prevention and protection measures to be adopted at the workplace. Sustainability 2020; 12:3603

11. Cockburn W: COVID-19: back to the workplace-Adapting workplaces and protecting workers. Europe. European Agency for Safety and Health at Work 2020; 1-8

12. Cristani M, Del Bue A, Murino V, Setti F \& Vinciarelli A: The Visual Social Distancing Problem. arXiv preprint arXiv 2020; 2005.04813

13. Ćosić K, Popović S, Šarlija $M$ \& Kesedžić I: Impact of human disasters and Covid-19 pandemic on mental health: Potential of digital psychiatry. Psychiatr Danub 2020; 32:25-31

14. Durand M J, Corbière M, Coutu MF, Reinharz D \& Albert $V$ : A review of best work-absence management and returnto-work practices for workers with musculoskeletal or common mental disorders. Work 2014; 48:579-589

15. El Husseiny A \& El Husseiny A: Redefining static and dynamic spaces genius integration in historic Cairo. Asian Journal of Environment-Behaviour Studies 2013; 4:133-146

16. Fischer R, Karl J, Bortolini T, Zilberberg M, Robinson K, Rabelo et al.: Rapid review and meta-meta-analysis of self-guided interventions to address anxiety, depression and stress during COVID-19 social distancing. PsyArXiv 2020;1- 33

17. Galea S, Merchant RM \& Lurie N: The mental health consequences of COVID-19 and physical distancing: The need for prevention and early intervention. JAMA Intern Med 2020; 180:817-818

18. Gupta DD \& Wong DW: No more "social distancing" but practice physical separation. Can J Public Health 2020; 111:488-489

19. Hamouche S: COVID-19 and employees' mental health: stressors, moderators and agenda for organizational actions. Emerald Open Research 2020; 2:1-15

20. Hecker S:Hazard Pay for COVID-19? Yes, But It's Not a Substitute for a Living Wage and Enforceable Worker Protections. New Solut 2020; 30:95-101

21. Jakovljevic M: Covid-19 crisis as a collective hero's journey to better public and global mental health. Psychiatr Danub 2020; 32:3-5

22. Jakovljevic $M$, Bjedov $S$, Jaksic $N$ \& Jakovljevic I: COVID-19 pandemia and public and global mental health from the perspective of global health security. Psychiatr Danub 2020; 32:6-14

23. Jin Z, Zhao K, Xia Y, Chen R, Yu H, Tamutana TT et al.: Psychological responses to the Coronavirus Disease (COVID-19) outbreak. ChinaXiv 2020; 14:1-16

24. Mehta V: The new proxemics: COVID-19, social distancing, and sociable space. Journal of Urban Design 2020; 25:669-674

25. Mustajab D, Bauw A, Rasyid A, Irawan A, Akbar MA \& Hamid MA: Working From Home Phenomenon As an Effort to Prevent COVID-19 Attacks and Its Impacts on Work Productivity. TIJAB 2020; 4:13-21 
26. Prin $M \&$ Bartels $K$ : Social distancing: implications for the operating room in the face of COVID-19. Can $J$ Anaesth 2020; 67:789-797

27. Qiu J, Shen B, Zhao M, Wang Z, Xie B \& Xu Y: A nationwide survey of psychological distress among Chinese people in the COVID-19 epidemic: implications and policy recommendations. Gen Psychiatry 2020; 33:1-3

28. Rajkumar RP: Attachment Theory and Psychological Responses to the COVID-19 Pandemic: A Narrative Review. Psychiatr Danub 2020; 32:256-261

29. Regmi $K$ \& Lwin CM: Impact of social distancing measures for preventing coronavirus disease 2019 [COVID19]: A systematic review and meta-analysis protocol. MedRxiv 2020; 1-19

30. Rueda-Garrido JC, Vicente-Herrero MT, Del Campo MT, Reinoso-Barbero L, De La Hoz R E, Delclos, G. L et al.: Return to work guidelines for the COVID-19 pandemic. Occup Med (Lond) 2020; 70:300-305

31. Shaw WS, Main CJ, Findley PA, Collie A, Kristman VL \& Gross DP: Opening the Workplace After COVID-19: What Lessons Can be Learned from Return-to-Work Research? J Occup Rehabil 2020; 30:299-302

32. Tan $W$, Hao F, Mcintyre RS, Jiang L, Jiang $X$, Zhang $L$ et al.: Is returning to work during the COVID-19 pandemic stressful? A study on immediate mental health status and psychoneuroimmunity prevention measures of Chinese workforce. Brain Behav Immun Health 2020; 87:84-92

33. Venkatesh $A$ \& Edirappuli $S$ : Social distancing in COVID-19: what are the mental health implications? BMJ 2020; 369:1

34. Vieira CM, Franco OH, Restrepo CG \& Abel T: COVID19: The forgotten priorities of the pandemic. Maturitas 2020; 136:38-41

35. Wang C, Horby PW, Hayden FG \& Gao GF: A novel coronavirus outbreak of global health concern. The Lancet 2020; 395:470-473
36. WHO: Considerations for public health and social measures in the workplace in the context of COVID-19 [Online]. Geneva: World Health Organization 2020a. (Accessed 0706 2020). Available at: https://www.who.int/ publications/ i/item/considerations-for-public-health-and-socialmeasures-in-the-workplace-in-the-context-of-covid-19

37. WHO: Coronavirus disease (COVID-19): health and safety at the workplace [Online]. World Health Organization 2020b. (Accessed 07-06-2020). Available at: https://www.who.int/news-room/q-a-detail/q-a-tips-for-healthand-safety-at-the-workplace-in-the-context-of-covid-19

38. WHO: WHO Director-General's opening remarks at the media briefing on COVID-19 - 11 March 2020 [Online]. Geneva: World Health Organization 2020c. (Accessed 1603-2020). Available at: https://www.who.int/dg/ speeches/ detail/who-director-general-s-opening-remarks-at-themedia-briefing-on-covid-19-11-march-2020

39. Williams SN, Armitage CJ, Tampe $T \&$ Dienes K: Public perceptions and experiences of social distancing and social isolation during the COVID-19 pandemic: A UKbased focus group study. MedRxiv 2020; 1-27

40. Xiang YT, Yang Y, Li W, Zhang L, Zhang $Q$, Cheung T et al.: Timely mental health care for the 2019 novel coronavirus outbreak is urgently needed. Lancet Psychiatry 2020; 7:228-229

41. Yip PS \& Chau PH : Physical Distancing and Emotional Closeness Amidst COVID-19. Crisis 2020; 41:153-155

42. Zhang $J, W u W$, Zhao $X$ \& Zhang $W$ : Recommended psychological crisis intervention response to the 2019 novel coronavirus pneumonia outbreak in China: a model of West China Hospital. Precis Clin Med 2020a; 3:3-8

43. Zhang W, Gao F, Gross J, Shrum L \& Hayne H: How Does Social Distancing During COVID-19 Affect Negative Moods and Memory? PsyArXiv 2020b; 1-20

44. Zhu Z, Xu S, Wang H, Liu Z, Wu J, LI G et al.: COVID-19 in Wuhan: Immediate Psychological Impact on 5062 Health Workers. medRxiv 2020; 1-23

Correspondence:

Salima Hamouche

Faculty of Management, Canadian University Dubai

Dubai, UAE

E-mail: salima.hamouche@cud.ac.ae 